\title{
Exponentially stable solution of mathematical model of agents dynamics on time scales
}

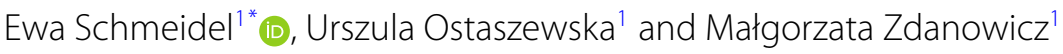

\section{"Correspondence:}

eschmeidel@math.uwb.edu.pl ${ }^{1}$ Institute of Mathematics, University of Bialystok, Białystok, Poland

\begin{abstract}
This work is an attempt at studying leader-following model on the arbitrary time scale. The step size is treated as a function of time. Our purpose is establishing conditions ensuring a leader-following consensus for any time scale basing on the Grönwall inequality. We give some examples illustrating the obtained results.

MSC: 34N05; 34D20; 93C10

Keywords: Time scales; Leader-following problem; Grönwall inequality; Multi-agent systems; Graph theory
\end{abstract}

The Author(s) 2019. This article is distributed under the terms of the Creative Commons Attribution 4.0 International License (http://creativecommons.org/licenses/by/4.0/), which permits unrestricted use, distribution, and reproduction in any medium, provided you give appropriate credit to the original author(s) and the source, provide a link to the Creative Commons license, and indicate if changes were made.

\section{Introduction}

The aim of this paper is to propose conditions ensuring the consensus of a multi-agent system over an arbitrary time scale. We consider continuous- and discrete-time models and also models on time scales simultaneously consisting of both kinds of points, right-dense and right-scattered. Under some assumptions, we prove that consensus can be achieved exponentially if the graininess function is bounded. All theorems are still true if the graininess function approaches zero. Some existing results of discrete-time consensus are particular cases of the results presented in this paper.

The leader-following problem has been investigated since 1970s. In 1974, DeGroot [1] studied an explicitly described model that resulted in the consensus. In 2000, Krause [2, 3] discussed the model of a group of agents who have to make a joint assessment of a certain magnitude. The coordination of groups of mobile autonomous agents based on the nearest-neighbor rules was considered by Jadbabaie et al. [4]. Blondel et al. [5, 6], took into account Krause's model with state-dependent connectivity. Girejko et al. [7, 8], examined Krause's model on discrete time scales. In 2007 there were published two important papers by Cucker and Smale $[9,10]$. The authors considered an emergent behavior in flocks. The Cucker-Smale model on isolated time scales was in the area of interests of Girejko et al. [8]. Girejko, Machado, Malinowska, and Martins [11] investigated sufficient conditions for consensus in the Cucker-Smale-type model on discrete time scales. In 2015, Wang et al. [12] published some results for the leader-following consensus of discrete-time linear multi-agent systems with communication noises.

inctich 
Our results generalize and improve the results obtained in [13] and [14]. In [14] consensus on different types of discrete-time scales is considered under the assumption that the feedback control gain $\gamma$ is constant.

\section{Basis of time scales calculus}

A time scale is a model of time [15-17], where the step size is a function of time. From mathematical point of view, it is an arbitrary nonempty closed subset $\mathbb{T}$ of the set $\mathbb{R}$ of real numbers.

The mapping $\sigma: \mathbb{T} \rightarrow \mathbb{T}$ defined by $\sigma(t)=\inf \{s \in \mathbb{T}: s>t\}$ with $\inf \emptyset=\sup \mathbb{T}$ is called the forward jump operator. Similarly, we define the backward jump operator $\rho: \mathbb{T} \rightarrow \mathbb{T}$ by $\rho(t)=\sup \{s \in \mathbb{T}: s<t\}$ with $\sup \emptyset=\inf \mathbb{T}$. The following classification of points is used within the theory: a point $t \in \mathbb{T}$ is called right-dense, right-scattered, left-dense, and leftscattered if $\sigma(t)=t$ (for $t<\sup \mathbb{T}), \sigma(t)>t, \rho(t)=t$ (for $t>\inf \mathbb{T}$ ), and $\rho(t)<t$, respectively. We say that $t$ is isolated if $\rho(t)<t<\sigma(t)$ and that $t$ is dense if $\rho(t)=t=\sigma(t)$. The function $\mu: \mathbb{T} \rightarrow[0, \infty)$ is defined by $\mu(t)=\sigma(t)-t$ and called the graininess function. The delta (or Hilger) derivative of $f: \mathbb{T} \rightarrow \mathbb{R}$ at a point $t \in \mathbb{T}^{\kappa}$, where

$$
\mathbb{T}^{\kappa}:= \begin{cases}\mathbb{T} \backslash(\rho(\sup \mathbb{T}), \sup \mathbb{T}] & \text { if } \sup \mathbb{T}<\infty, \\ \mathbb{T} & \text { if } \sup \mathbb{T}=\infty,\end{cases}
$$

is defined in the following way.

Definition 1 ([16]) The delta derivative $f^{\Delta}(t)$ is the number (provided that it exists) with the property that given any $\varepsilon>0$, there is a neighborhood $U$ of $t$ (i.e., $U=(t-\delta, t+\delta) \cap \mathbb{T}$ for some $\delta>0$ ) such that

$$
\left|(f(\sigma(t))-f(s))-f^{\Delta}(t)(\sigma(t)-s)\right| \leq \varepsilon|\sigma(t)-s| \quad \text { for all } s \in U \text {. }
$$

Moreover, we say that $f$ is delta (or Hilger) differentiable (or, shortly, differentiable) on $\mathbb{T}^{\kappa}$ if $f^{\Delta}(t)$ exists for all $t \in \mathbb{T}^{\kappa}$. Then the function $f^{\Delta}: \mathbb{T}^{\kappa} \rightarrow \mathbb{R}$ is called the (delta) derivative of $f$ on $\mathbb{T}^{\kappa}$.

The following definitions will be further used, too.

Definition 2 ([16]) A function $f: \mathbb{T} \rightarrow \mathbb{R}$ is called regulated if its right-sided limits exist (finite) at right-dense points in $\mathbb{T}$ and its left-sided limits exist (finite) at left-dense points in $\mathbb{T}$. A function $f: \mathbb{T} \rightarrow \mathbb{R}$ is called rd-continuous if it is continuous at right-dense points in $\mathbb{T}$ and its left-side limits exist (finite) at left-dense points in $\mathbb{T}$.

Definition 3 ([16]) Let $f: \mathbb{T} \rightarrow \mathbb{R}$ be a regulated function. We define the indefinite integral of $f$ by $\int f(t) \Delta t=F(t)+c$, where $c$ is an arbitrary constant, and $F$ is a preantiderivative of $f$. We define the Cauchy integral of $f$ as $\int_{a}^{b} f(t) \Delta t=F(b)-F(a)$ for all $a, b \in \mathbb{T}$.

Definition 4 ([16]) We say that a function $p: \mathbb{T} \rightarrow \mathbb{R}$ is regressive if $1+\mu(t) p(t) \neq 0$ for all $t \in \mathbb{T}^{\kappa}$. The set of all regressive and rd-continuous functions $p: \mathbb{T} \rightarrow \mathbb{R}$ is denoted by $\mathcal{R}$. The set of all positively regressive elements of $\mathcal{R}$ is defined as $\mathcal{R}^{+}:=\{p \in \mathcal{R}: 1+\mu(t) p(t)>$ 0 for all $t \in \mathbb{T}\}$. 
Definition 5 ([16]) An $N \times N$-matrix-valued function $P$ on a time scale $\mathbb{T}$ is called regressive (with respect to $\mathbb{T}$ ) if

$$
I+\mu(t) P(t) \quad \text { is invertible for all } t \in \mathbb{T}^{\kappa},
$$

where by $I$ we denote the $N \times N$ identity matrix.

Similarly to the scalar case, the class of all regressive and rd-continuous matrix-valued functions is denoted by $\mathcal{R}$. Notice that a constant $N \times N$ matrix $P$ is regressive iff the eigenvalues $\lambda_{i}$ of $P$ are regressive for all $1 \leq i \leq N$.

We use the Grönwall inequality in the proof of the main result.

Lemma 1 ([16]) Let $z$ be rd-continuous, $p \in \mathcal{R}^{+}$, and $p(t) \geq 0$ for $t \in \mathbb{T}$ and $c \in \mathbb{R}$. Then

$$
z(t) \leq c+\int_{T_{0}}^{t} p(\tau) z(\tau) \Delta \tau \quad \text { for all } t \in \mathbb{T}
$$

implies

$$
z(t) \leq c e_{p}\left(t, T_{0}\right)
$$

Here $z(t)=e_{p}\left(t, T_{0}\right), T_{0} \in \mathbb{T}$, is a solution of the initial value problem

$$
z^{\Delta}(t)=p(t) z(t), \quad z\left(T_{0}\right)=1 \quad \text { on } \mathbb{T} .
$$

We assume that

$$
\inf \mathbb{T}=T_{0} \geq 0 \quad \text { and } \quad \sup \mathbb{T}=\infty
$$

which implies that $\mathbb{T}^{\kappa}=\mathbb{T}$.

\section{Mathematical model of agents dynamics}

We consider a multi-agent system consisting of $N$ agents and the leader. The dynamics of each agent labeled $i, i=1,2, \ldots, N$, is given by the following equation:

$$
x_{i}^{\Delta}(t)=f\left(t, x_{i}(t)\right)+\gamma(t) \sum_{j=1}^{N} a_{i j}\left(x_{j}(t)-x_{i}(t)\right)+\gamma(t) d_{i}\left(x_{0}(t)-x_{i}(t)\right),
$$

where $t \in \mathbb{T}, x_{i}: \mathbb{T} \rightarrow \mathbb{R}$, and $\gamma: \mathbb{T} \rightarrow \mathbb{R}$ represent the state and the feedback control gain at time $t$, respectively. Here $a_{i j} \in \mathbb{R}, d_{i} \in \mathbb{R}, i, j=1,2, \ldots, N$, and $D:=\operatorname{diag}\left[d_{1}, d_{2}, \ldots, d_{N}\right]$ is a diagonal matrix. Throughout this paper, we assume that $a_{i j}=a_{j i}$, which means that the matrix $A=\left[a_{i j}\right]_{N \times N}$ is symmetric. The function $f: \mathbb{T} \times \mathbb{R} \rightarrow \mathbb{R}$ describes nonlinear dynamics. The leader, labeled as $i=0$, for multi-agent system (2) is an isolated agent with trajectory described by

$$
x_{0}^{\Delta}(t)=f\left(t, x_{0}(t)\right), \quad t \in \mathbb{T} .
$$


Notice that the control law $\gamma(t) \sum_{j=1}^{N} a_{i j}\left(x_{j}(t)-x_{i}(t)\right)+\gamma(t) d_{i}\left(x_{0}(t)-x_{i}(t)\right)$ for the $i$ th agent used in system (2)-(3) was studied by many authors, including Yu, Jiang, and $\mathrm{Hu}$ [18].

Let $\varepsilon_{i}(t)=x_{i}(t)-x_{0}(t)$ denote the distance between the leader and the $i$ th agent. From (2)-(3) we obtain

$$
\varepsilon_{i}^{\Delta}(t)=f\left(t, x_{i}(t)\right)-f\left(t, x_{0}(t)\right)+\gamma(t) \sum_{j=1}^{N} a_{i j}\left(\varepsilon_{j}(t)-\varepsilon_{i}(t)\right)-\gamma(t) d_{i} \varepsilon_{i}(t)
$$

for $i=1,2, \ldots, N$. Setting

$$
\begin{aligned}
& \varepsilon(t)=\left(\varepsilon_{1}(t), \varepsilon_{2}(t), \ldots, \varepsilon_{N}(t)\right)^{T}, \\
& x(t)=\left(x_{1}(t), x_{2}(t), \ldots, x_{N}(t)\right)^{T},
\end{aligned}
$$

and

$$
\begin{aligned}
& F(t, x(t))=\left(f\left(t, x_{1}(t)\right), f\left(t, x_{2}(t)\right), \ldots, f\left(t, x_{N}(t)\right)\right)^{T}, \\
& F\left(t, x_{0}(t) \mathbb{1}\right)=\left(f\left(t, x_{0}(t)\right), f\left(t, x_{0}(t)\right), \ldots, f\left(t, x_{0}(t)\right)\right)^{T},
\end{aligned}
$$

system (2)-(3) takes the following form:

$$
\varepsilon^{\Delta}(t)=F(t, x(t))-F\left(t, x_{0}(t) \mathbb{1}\right)-\gamma(t) B \varepsilon(t), \quad \varepsilon\left(T_{0}\right)=\varepsilon_{T_{0}}
$$

(for details, see [19]). Here $\mathbb{1}$ is the vector $(1, \ldots, 1)^{T}$. Recall that $B=L+D$ is a symmetric matrix since $A$ is a symmetric matrix and $L$ is the Laplacian matrix $L=\left[l_{i j}\right]$ with $l_{i i}=\sum_{j \neq i} a_{i j}$ and $l_{i j}=-a_{i j}, i, j=1, \ldots, N, i \neq j$.

If

$$
(-\gamma(t) B) \in \mathcal{R}
$$

in equation (1), then by $e_{-\gamma B}\left(t, T_{0}\right)$ we denote a solution of the initial value problem

$$
\varepsilon^{\Delta}(t)=-\gamma(t) B \varepsilon(t), \quad \varepsilon\left(T_{0}\right)=\mathbb{1} .
$$

By variation of constants (see [16]) the unique solution of equation (5) is given by

$$
\varepsilon(t)=e_{-\gamma B}\left(t, T_{0}\right) \varepsilon_{T_{0}}+\int_{T_{0}}^{t} e_{-\gamma B}(t, \sigma(\tau))\left(F\left(\tau, x_{0}(\tau) \mathbb{1}\right)-F(\tau, x(\tau))\right) \Delta \tau
$$

Definition 6 A function $F: \mathbb{T} \times \mathbb{R}^{N} \rightarrow \mathbb{R}^{N}$ fulfills Lipschitz condition with respect to the second variable if there exists a positive constant $\mathcal{L}$ such that

$$
\|F(t, x)-F(t, y)\| \leq \mathcal{L}\|x-y\|, \quad t \in \mathbb{T}
$$

Definition 7 We say that equation (5), where $T_{0} \geq 0, \varepsilon_{T_{0}} \in \mathbb{R}^{N}$, is exponentially stable if there exist positive constants $c$ and $d$ such that, for any rd-continuous solution $\varepsilon\left(t, T_{0}, \varepsilon_{T_{0}}\right)$ of equation (5), we have

$$
\lim _{t \rightarrow \infty}\left\|\varepsilon\left(t, T_{0}, \varepsilon_{T_{0}}\right)\right\|=: \lim _{t \rightarrow \infty}\|\varepsilon(t)\| \leq c\left\|\varepsilon_{T_{0}}\right\| \lim _{t \rightarrow \infty} e_{d}\left(t, T_{0}\right)=0
$$


For some relevant result on the exponential stability in the discrete case, see [20] and [21].

Definition 8 The multi-agent system (2)-(3) is said to achieve the leader-following consensus exponentially if equation (5) is exponentially stable.

In 2005, Peterson and Raffoul [22] investigated the exponential stability of the zero solution to systems of dynamic equations on time scales. The authors defined suitable Lyapunov-type functions and then formulated appropriate inequalities on these functions that guarantee that the zero solution exponentially decays to zero. For the growth of generalized exponential functions on time scales, see Bodine and Lutz [23].

\section{Main results}

Assume that the function $F: \mathbb{T} \times \mathbb{R}^{N} \rightarrow \mathbb{R}^{N}$ defined by (4) satisfies Lipschitz condition with respect to the second variable.

Let $\lambda_{i}, i=1,2, \ldots, N$, be the eigenvalues of the matrix $B$. By $\mathbb{T}^{s}$ and $\mathbb{T}^{d}$ we denote the sets of right-scattered and right-dense points of $\mathbb{T}$, respectively. Notice that since by assumption $\sup \mathbb{T}=\infty$, at least one of the sets $\mathbb{T}^{s}$ or $\mathbb{T}^{d}$ must be unbounded.

Next, we rewrite the time scale $\mathbb{T}$ in a useful way for estimation of the norm of a solution of the initial value problem (5) on a time scale consisting of right-scattered and rightdense points. To avoid confusion, we underline that any interval throughout this paper is an interval on the time scale, that is, any interval contains only points of the time scale. Set

$$
\begin{aligned}
& T_{1}=\min \left\{t: t \in\left[T_{0}, \infty\right) \cap \mathbb{T}^{d} \text { and }\left[T_{0}, t\right) \subset \mathbb{T}^{s}\right\}, \\
& T_{2 i}=\inf \left\{t: t \in\left[T_{2 i-1}, \infty\right) \cap \mathbb{T}^{s} \text { and }\left[T_{2 i-1}, t\right) \subset \mathbb{T}^{d}\right\} \\
& T_{2 i+1}=\min \left\{t: t \in\left[T_{2 i}, \infty\right) \cap \mathbb{T}^{d} \text { and }\left[T_{2 i}, t\right) \subset \mathbb{T}^{s}\right\}
\end{aligned}
$$

for $i=1,2, \ldots$ In case of $\left[T_{2 i-1}, \infty\right) \cap \mathbb{T}^{s}=\emptyset$ for some $i \in \mathbb{N}$ we take $T_{2 i}=\infty$ (see Example 9). If $\left[T_{2 i-1}, t\right) \cap \mathbb{T}^{d}=\left[T_{2 i-1}, T_{2 i-1}\right)=\emptyset$ for some $i \in \mathbb{N}$, then we also take $T_{2 i}=\infty$ (see Example 6). Analogously, if $\left[T_{2 i}, \infty\right) \cap \mathbb{T}^{d}=\emptyset$ for some $i \in \mathbb{N}$, then $T_{2 i+1}=\infty$. If $T_{j}=\infty$ for some $j \in \mathbb{N}_{0}$, then we take $T_{j+i}=T_{j}$ for $i \in \mathbb{N}$ and $\left[T_{j+i}, T_{j+i+1}\right)=\left(T_{j+i}, T_{j+i+1}\right]=\emptyset$ (see Example 7). We see that if $\sigma\left(T_{0}\right)=T_{0}$, then $T_{1}=T_{0}$.

Example 1 Let $\mathbb{T}=\{1\} \cup[2,3] \cup[6, \infty)$. Here $T_{0}=1, T_{1}=2, T_{2}=3, T_{3}=6$, and $T_{4}=\infty$.

We underline that $T_{2 i+1} \in \mathbb{T}^{d}$ for any $i \in \mathbb{N}_{0}$, but it is possible that $T_{2 i} \notin \mathbb{T}^{s}$ for some $i \in \mathbb{N}_{0}$.

Example 2 Let $\mathbb{T}=\bigcup_{i=1}^{\infty}[2 i-1,2 i] \cup\left\{4 i+\frac{1}{j+1}: i, j \in \mathbb{N}\right\}$. Here $T_{0}=1, T_{1}=T_{0}$, and $T_{i}=i$ for $i \in\{2,3, \ldots\}$. We see that $T_{1}=T_{0} \in \mathbb{T}^{d}, T_{2 i+1} \in \mathbb{T}^{d}, T_{4 i} \in \mathbb{T}^{d}$, and $T_{4 i+2} \in \mathbb{T}^{s}$ for $i \in \mathbb{N}$.

Example 3 Let $\mathbb{T}=\bigcup_{i=1}^{\infty}[2 i-1,2 i] \cup\left\{4 i+1-\frac{1}{j+1}: i, j \in \mathbb{N}\right\}$. Here $T_{0}=1, T_{i}=i$ for $i \in \mathbb{N}$, and $T_{2 i-1} \in \mathbb{T}^{d}$ and $T_{2 i} \in \mathbb{T}^{s}$ for $i \in \mathbb{N}$. 
We can write

$$
\mathbb{T}=\left\{T_{0}\right\} \cup \bigcup_{j=0}^{\infty}\left(T_{j}, T_{j+1}\right]=\left\{T_{0}\right\} \cup \bigcup_{i=0}^{\infty}\left(T_{2 i}, T_{2 i+1}\right] \cup\left(T_{2 i+1}, T_{2 i+2}\right]
$$

wherein $\left(T_{2 i}, T_{2 i+1}\right) \subset \mathbb{T}^{s}$ and $\left(T_{2 i+1}, T_{2 i+2}\right) \subset \mathbb{T}^{d}$.

In the next lemma, for any $i \in \mathbb{N}$, we present the estimates of the norms of matrices $e_{-\gamma B}\left(t, T_{2 i}\right)$ for $t \in\left[T_{2 i}, T_{2 i+1}\right)$ and $e_{-\gamma B}\left(t, T_{2 i+1}\right)$ for $t \in\left[T_{2 i+1}, T_{2 i+2}\right)$.

Lemma 2 Suppose that, for $i=1,2, \ldots, N$, the following conditions are satisfied:

$$
\begin{aligned}
& \gamma(t) \lambda_{i} \in(0, \infty) \quad \text { for } t \in \mathbb{T}, \\
& 0<\delta \leq \mu(t) \gamma(t) \lambda_{i}<1 \quad \text { for } t \in \mathbb{T}^{s}, \text { where } \delta \text { is a constant. }
\end{aligned}
$$

Then there exists a positive real number $\mathcal{M}<1$ such that

$$
\begin{aligned}
& \left\|e_{-\gamma B}\left(t, T_{2 i}\right)\right\| \leq \prod_{s \in\left[T_{2 i}, t\right)} \mathcal{M} \text { for } t \in\left[T_{2 i}, T_{2 i+1}\right), i \in \mathbb{N}_{0}, \\
& \left\|e_{-\gamma B}\left(t, T_{2 i+1}\right)\right\| \leq \mathcal{M}^{\int_{2 i+1}^{t}|\gamma(s)| d s} \text { for } t \in\left[T_{2 i+1}, T_{2 i+2}\right), i \in \mathbb{N}_{0},
\end{aligned}
$$

where $\|\cdot\|$ denotes the spectral norm of the considered matrix at the point $t$.

Proof Obviously, $\mathbb{T}^{s} \cup \mathbb{T}^{d}=\mathbb{T}$. We consider two cases:

(i) $t \in \mathbb{T}^{s}$;

(ii) $t \in \mathbb{T}^{d}$.

In case (i), notice that since matrix $B$ is symmetric, $I-\mu(t) \gamma(t) B$ is also a symmetric matrix at the point $t$. Therefore $\|I-\mu(t) \gamma(t) B\|$ equals the maximum of the absolute value of eigenvalues of the matrix $I-\mu(t) \gamma(t) B$. This means that

$$
\left\|e_{-\gamma B}\left(t, T_{2 i}\right)\right\|=\prod_{s \in\left(T_{2 i}, t\right]}\|I-\mu(s) \gamma(s) B\|=\prod_{s \in\left(T_{2 i}, t\right]}\left(\max _{i \in\{1,2, \ldots, N\}}\left\{\left|1-\mu(s) \gamma(s) \lambda_{i}\right|\right\}\right)
$$

for $t \in\left[T_{2 i}, T_{2 i+1}\right)$. Because of the positivity of $\mu$ on $\mathbb{T}^{s}$ and condition (8), we have $\left|\mu(s) \gamma(s) \lambda_{i}\right|=\mu(s) \gamma(s) \lambda_{i}$. Moreover, by $(9), \mu(s) \gamma(s) \lambda_{i} \in(0,1)$ for $i \in\{1,2, \ldots, N\}$. We can conclude

$$
\left\|e_{-\gamma B}\left(t, T_{2 i}\right)\right\|=\prod_{s \in\left(T_{2 i}, t\right]}\left(1-\min _{i \in\{1,2, . ., N\}}\left\{\mu(s) \gamma(s) \lambda_{i}\right\}\right) .
$$

Again by (9), we have

$$
-1<-\min _{i \in\{1,2, . ., N\}} \mu(s) \gamma(s) \lambda_{i} \leq-\delta<0 .
$$

By the preceding we have

$$
\left\|e_{-\gamma B}\left(t, T_{2 i}\right)\right\| \leq \prod_{s \in\left(T_{2 i}, t\right]}(1-\delta)=\prod_{s \in\left(T_{2 i}, t\right]} \mathcal{M}^{*}=\prod_{s \in\left[T_{2 i}, t\right)} \mathcal{M}^{*} \text { for } t \in\left[T_{2 i}, T_{2 i+1}\right),
$$

where $\mathcal{M}^{*}:=1-\delta \in(0,1)$. 
Case (ii). Condition (8) implies

$\left(1^{\circ}\right) \lambda_{i}>0$ for any $i=1,2, \ldots, N$ and $\gamma(t)>0$ for any $t \in \mathbb{T}^{d}$

or

$\left(2^{\circ}\right) \quad \lambda_{i}<0$ for any $i=1,2, \ldots, N$ and $\gamma(t)<0$ for any $t \in \mathbb{T}^{d}$.

If $\left(1^{\circ}\right)$, then

$$
\begin{aligned}
\left\|e_{-\gamma B}\left(t, T_{2 i+1}\right)\right\| & =\left\|e^{B}\right\|^{-\int_{T_{2 i+1}}^{t} \gamma(s) d s}=\left(\max _{i \in\{1,2, \ldots, N\}}\left\{e^{\lambda_{i}}\right\}\right)^{-\int_{T_{2 i+1}}^{t} \gamma(s) d s} \\
& =\left(\mathcal{M}^{* *}\right)^{\int_{T_{2 i+1}}^{t} \gamma(s) d s}=\left(\mathcal{M}^{* *}\right)^{\int_{T_{2 i+1}}^{t}|\gamma(s)| d s}
\end{aligned}
$$

for $t \in\left[T_{2 i+1}, T_{2 i+2}\right)$, where $\mathcal{M}^{* *}:=\left(\max _{i \in\{1,2, \ldots, N\}}\left\{e^{\lambda_{i}}\right\}\right)^{-1} \in(0,1)$.

If $\left(2^{\circ}\right)$, then

$$
\begin{aligned}
\left\|e_{-\gamma B}\left(t, T_{2 i+1}\right)\right\| & =\left\|e^{B}\right\|^{-\int_{T_{2 i+1}}^{t} \gamma(s) d s}=\left(\max _{i \in\{1,2, \ldots, N\}}\left\{e^{\lambda_{i}}\right\}\right)^{-\int_{T_{2 i+1}}^{t} \gamma(s) d s} \\
& =\left(\mathcal{M}^{* *}\right)^{-\int_{T_{2 i+1}}^{t} \gamma(s) d s}=\left(\mathcal{M}^{* *}\right)^{\int_{T_{2 i+1}}^{t}|\gamma(s)| d s}
\end{aligned}
$$

for $t \in\left[T_{2 i+1}, T_{2 i+2}\right)$, where $\mathcal{M}^{* *}:=\max _{i \in\{1,2, \ldots, N\}}\left\{e^{\lambda_{i}}\right\} \in(0,1)$.

Set $\mathcal{M}:=\max \left\{\mathcal{M}^{*}, \mathcal{M}^{* *}\right\}$. Obviously $\mathcal{M} \in(0,1)$.

Hence $\left\|e_{-\gamma B}\left(t, T_{2 i}\right)\right\| \leq \prod_{s \in\left[T_{2 i}, t\right)} \mathcal{M}$ for $t \in\left[T_{2 i}, T_{2 i+1}\right)$ and $\left\|e_{-\gamma B}\left(t, T_{2 i+1}\right)\right\| \leq$ $\mathcal{M}^{\int_{T 2 i+1}^{t}|\gamma(s)| d s}$ for $t \in\left[T_{2 i+1}, T_{2 i+2}\right)$.

Next, we find the estimates of the norm of the matrix $e_{-\gamma B}\left(t, T_{0}\right)$ in two cases, $t \in$ $\left[T_{2 i}, T_{2 i+1}\right)$ and $t \in\left[T_{2 i+1}, T_{2 i+2}\right)$.

Lemma 3 If conditions (8)-(9) are satisfied, then

$$
\left\|e_{-\gamma B}\left(t, T_{0}\right)\right\| \leq\left(\mathcal{M}^{\sum_{j=1}^{i} \int_{T_{2 j-1}}^{T_{2 j}}|\gamma(s)| d s}\right)\left(\prod_{s \in\left[T_{0}, t\right) \cap \mathbb{T}^{s}} \mathcal{M}\right)
$$

for $t \in\left[T_{2 i}, T_{2 i+1}\right)$, and

$$
\left\|e_{-\gamma B}\left(t, T_{0}\right)\right\| \leq\left(\prod_{s \in\left[T_{0}, T_{2 i+1}\right) \cap \mathbb{T}^{s}} \mathcal{M}\right)\left(\mathcal{M}^{\sum_{j=1}^{i} \int_{T_{2 j-1}}^{T_{2 j}}|\gamma(s)| d s+\int_{T_{2 i+1}}^{t}|\gamma(s)| d s}\right)
$$

for $t \in\left[T_{2 i+1}, T_{2 i+2}\right)$, where $i \in \mathbb{N}_{0}$.

Proof Let us rewrite the function $e_{-\gamma B}\left(t, T_{0}\right)$ in the form

$$
\begin{aligned}
e_{-\gamma B}\left(t, T_{0}\right)= & \left(\prod_{s \in\left[T_{0}, T_{1}\right)}(I-\mu(s) \gamma(s) B)\right)\left(e^{-B \int_{T_{1}}^{T_{2}} \gamma(s) d s}\right) \\
& \cdot\left(\prod_{s \in\left[T_{2}, T_{3}\right)}(I-\mu(s) \gamma(s) B)\right)\left(e^{-B \int_{T_{3}}^{T_{4}} \gamma(s) d s}\right) \\
& \ldots
\end{aligned}
$$




$$
\begin{aligned}
& \cdot\left(\prod_{s \in\left[T_{2 i-2}, T_{2 i-1}\right)}(I-\mu(s) \gamma(s) B)\right)\left(e^{-B \int_{T_{2 i-1}}^{T_{2 i}} \gamma(s) d s}\right) \\
& \cdot\left(\prod_{s \in\left[T_{2 i}, t\right]}(I-\mu(s) \gamma(s) B)\right) \text { for } t \in\left[T_{2 i}, T_{2 i+1}\right)
\end{aligned}
$$

or

$$
\begin{aligned}
e_{-\gamma B}\left(t, T_{0}\right)= & \left(\prod_{s \in\left[T_{0}, T_{1}\right)}(I-\mu(s) \gamma(s) B)\right)\left(e^{-B \int_{T_{1}}^{T_{2}} \gamma(s) d s}\right) \\
& \cdot\left(\prod_{s \in\left[T_{2}, T_{3}\right)}(I-\mu(s) \gamma(s) B)\right)\left(e^{-B \int_{T_{3}}^{T_{4}} \gamma(s) d s}\right) \\
& \ldots \\
& \cdot\left(\prod_{s \in\left[T_{2 i-2}, T_{2 i-1}\right)}(I-\mu(s) \gamma(s) B)\right)\left(e^{-B \int_{T_{2 i-1}}^{T_{2 i}} \gamma(s) d s}\right) \\
& \cdot\left(\prod_{s \in\left[T_{2 i}, T_{2 i+1)]}\right.}(I-\mu(s) \gamma(s) B)\right)\left(e^{-B \int_{T_{2 i+1}}^{t} \gamma(s) d s}\right) \quad \text { for } t \in\left[T_{2 i+1}, T_{2 i+2}\right) .
\end{aligned}
$$

By submultiplicativity of the norm, for $t \in\left[T_{2 i}, T_{2 i+1}\right)$, we estimate the norm of the matrix $e_{-\gamma B}\left(t, T_{0}\right)$ :

$$
\begin{aligned}
& \left\|e_{-\gamma B}\left(t, T_{0}\right)\right\| \leq\left(\prod_{s \in\left[T_{0}, T_{1}\right)}\|I-\mu(s) \gamma(s) B\|\right)\left(\left\|e^{B}\right\|^{-\int_{T_{1}}^{T_{2}}|\gamma(s)| d s}\right) \\
& \cdot\left(\prod_{s \in\left[T_{2}, T_{3}\right)}\|I-\mu(s) \gamma(s) B\|\right)\left(\left\|e^{B}\right\|^{-\int_{T_{3}}^{T_{4}}|\gamma(s)| d s}\right) \\
& \text {... } \\
& \cdot\left(\prod_{s \in\left[T_{2 i-2}, T_{2 i-1}\right)}\|I-\mu(s) \gamma(s) B\|\right)\left(\left\|e^{B}\right\|^{-\int_{T_{2 i-1}}^{T_{2 i}}|\gamma(s)| d s}\right) \\
& \cdot\left(\prod_{s \in\left[T_{2 i}, t\right)}\|I-\mu(s) \gamma(s) B\|\right) \\
& \leq\left(\prod_{s \in\left[T_{0}, T_{1}\right)} \mathcal{M}\right)\left(\mathcal{M}^{\int_{T_{1}}^{T_{2}}|\gamma(s)| d s}\right)\left(\prod_{s \in\left[T_{2}, T_{3}\right)} \mathcal{M}\right)\left(\mathcal{M}^{\int_{T_{3}}^{T_{4}}|\gamma(s)| d s}\right) \cdots \\
& \cdot\left(\mathcal{M}^{\int_{T_{2 i-1}}^{T_{2 i}}|\gamma(s)| d s}\right)\left(\prod_{s \in\left[T_{2 i}, t\right)} \mathcal{M}\right) \\
& =\left(\prod_{s \in\left[T_{0}, t\right) \cap \mathbb{T}^{s}} \mathcal{M}\right)\left(\mathcal{M}^{\int_{T_{1}}^{T_{2}}|\gamma(s)| d s+\int_{T_{3}}^{T_{4}}|\gamma(s)| d s+\cdots+\int_{T_{2 i-1}}^{T_{2 i}}|\gamma(s)| d s}\right) \\
& =\left(\mathcal{M}^{\sum_{j=1}^{i} \int_{T_{2 j-1}}^{T_{2 j}}|\gamma(s)| d s}\right)\left(\prod_{s \in\left[T_{0}, t\right) \cap \mathbb{T}^{s}} \mathcal{M}\right) .
\end{aligned}
$$

Analogously, for $t \in\left[T_{2 i+1}, T_{2 i+2}\right)$, we get

$$
\left\|e_{-\gamma B}\left(t, T_{0}\right)\right\| \leq\left(\prod_{s \in\left[T_{0}, T_{2 i+1}\right) \cap \mathbb{T}^{s}} \mathcal{M}\right)\left(\mathcal{M}^{\sum_{j=1}^{i} \int_{T_{2 j-1}}^{T_{2 j}}|\gamma(s)| d s+\int_{T_{2 i+1}}^{t}|\gamma(s)| d s}\right) .
$$


Remark 1 If conditions (8)-(9) are satisfied, then

$$
\left\|e_{-\gamma B}\left(t, T_{0}\right)\right\| \leq \prod_{s \in\left[T_{0}, t\right) \cap \mathbb{T}^{s}} \mathcal{M} \text { for } t \in \mathbb{T}
$$

Proof Since $\mathcal{M} \in(0,1)$ and $\sum_{j=1}^{i} \int_{T_{2 j-1}}^{T_{2 j}}|\gamma(s)| d s+\int_{T_{2 i+1}}^{t}|\gamma(s)| d s \geq 0$, we get

$$
\mathcal{M}^{\sum_{j=1}^{i} \int_{T_{2 j-1}}^{T_{2 j}}|\gamma(s)| d s+\int_{T_{2 i+1}}^{t}|\gamma(s)| d s} \leq 1 \quad \text { for } t \in \mathbb{T} .
$$

From the above, inequalities (10) and (11) imply

$$
\begin{aligned}
& \left\|e_{-\gamma B}\left(t, T_{0}\right)\right\| \leq \prod_{s \in\left[T_{0}, t\right) \cap \mathbb{T}^{s}} \mathcal{M} \text { for } t \in\left[T_{2 i}, T_{2 i+1}\right), \\
& \left\|e_{-\gamma B}\left(t, T_{0}\right)\right\| \leq \prod_{s \in\left[T_{0}, T_{2 i+1}\right) \cap \mathbb{T}^{s}} \mathcal{M} \text { for } t \in\left[T_{2 i+1}, T_{2 i+2}\right),
\end{aligned}
$$

where $i \in \mathbb{N}_{0}$, and this is our claim.

The following result concerns the scalar case of exponential function on arbitrary time scale.

Lemma 4 Let $e_{\mathcal{L M}^{-1}\left(\cdot, T_{0}\right): \mathbb{T} \rightarrow \mathbb{R} \text {. Then }}$

$$
e_{\mathcal{L M}^{-1}}\left(t, T_{0}\right)=e^{\mathcal{L M}^{-1} \sum_{j=1}^{i}\left(T_{2 j}-T_{2 j-1}\right)} \prod_{s \in\left[T_{0}, t\right) \cap \mathbb{T}^{s}}\left(1+\mu(s) \mathcal{L} \mathcal{M}^{-1}\right)
$$

for $t \in\left[T_{2 i}, T_{2 i+1}\right)$, and

$$
e_{\mathcal{L} \mathcal{M}^{-1}}\left(t, T_{0}\right)=\prod_{s \in\left[T_{0}, T_{2 i+1}\right) \cap \mathbb{T}^{s}}\left(1+\mu(s) \mathcal{L} \mathcal{M}^{-1}\right) \cdot e^{\mathcal{L} \mathcal{M}^{-1}\left(\sum_{j=1}^{i}\left(T_{2 j}-T_{2 j-1}\right)+\left(t-T_{2 j+1}\right)\right)}
$$

for $t \in\left[T_{2 i+1}, T_{2 i+2}\right)$, where $i \in \mathbb{N}_{0}$.

We are now in a position to present the main theorem of this paper.

Theorem 1 Suppose that conditions (7)-(9) are satisfied and for any $t \in \mathbb{T}$,

there exists a positive constant $\mu^{*}$ such that $\mu(t) \leq \mu^{*}$,

$$
\lim _{t \rightarrow \infty} e^{\mathcal{L} \mathcal{M}^{-1} \sum_{j=1}^{i}\left(T_{2 j}-T_{2 j-1}\right)} \mathcal{M}^{\sum_{j=1}^{i} \int_{T_{2 j-1}}^{T_{2 j}}|\gamma(s)| d s} \prod_{s \in\left[T_{0}, t\right) \cap \mathbb{T}^{s}}\left(\mathcal{M}+\mu^{*} \mathcal{L}\right)=0,
$$

and

$$
\begin{aligned}
& \lim _{t \rightarrow \infty} e^{\mathcal{L M}^{-1}\left(\sum_{j=1}^{i}\left(T_{2 j}-T_{2 j-1}\right)+\left(t-T_{2 j+1}\right)\right)} \\
& \cdot \mathcal{M}^{\sum_{j=1}^{i} \int_{T_{2 j-1}}^{T_{2 j}}|\gamma(s)| d s+\int_{T_{2 i+1}}^{t}|\gamma(s)| d s} \prod_{s \in\left[T_{0}, T_{2 i+1}\right) \cap \mathbb{T}^{s}}\left(\mathcal{M}+\mu^{*} \mathcal{L}\right)=0 .
\end{aligned}
$$

Then equation (5) is exponentially stable. 
Proof Taking the norm of the both sides of equation (6), we obtain

$$
\|\varepsilon(t)\|=\left\|e_{-\gamma B}\left(t, T_{0}\right) \varepsilon_{T_{0}}+\int_{T_{0}}^{t} e_{-\gamma B}(t, \sigma(\tau))\left(F\left(\tau, x_{0}(\tau) \mathbb{1}\right)-F(\tau, x(\tau))\right) \Delta \tau\right\| .
$$

Using properties of the norm, we get

$$
\|\varepsilon(t)\| \leq\left\|e_{-\gamma B}\left(t, T_{0}\right)\right\|\left\|\varepsilon_{T_{0}}\right\|+\left\|\int_{T_{0}}^{t} e_{-\gamma B}(t, \sigma(\tau))\left(F\left(\tau, x_{0}(\tau) \mathbb{1}\right)-F(\tau, x(\tau))\right) \Delta \tau\right\|,
$$

and consequently

$$
\|\varepsilon(t)\| \leq\left\|\varepsilon_{T_{0}}\right\|\left\|e_{-\gamma B}\left(t, T_{0}\right)\right\|+\int_{T_{0}}^{t}\left\|e_{-\gamma B}(t, \sigma(\tau))\right\|\left\|\left(F\left(\tau, x_{0}(\tau) \mathbb{1}\right)-F(\tau, x(\tau))\right)\right\| \Delta \tau .
$$

By condition (7) we obtain

$$
\|\varepsilon(t)\| \leq\left\|\varepsilon_{T_{0}}\right\|\left\|e_{-\gamma B}\left(t, T_{0}\right)\right\|+\mathcal{L} \int_{T_{0}}^{t}\left\|e_{-\gamma B}(t, \sigma(\tau))\right\|\|\varepsilon(\tau)\| \Delta \tau
$$

For $t \in\left[T_{2 i}, T_{2 i+1}\right)$, using (10), we estimate

$$
\begin{aligned}
\|\varepsilon(t)\| \leq & \left\|\varepsilon_{T_{0}}\right\|\left(\mathcal{M}^{\sum_{j=1}^{i} \int_{T_{2 j-1}}^{T_{2 j}}|\gamma(s)| d s}\right) \prod_{s \in\left[T_{0}, t\right) \cap \mathbb{T}^{s}} \mathcal{M} \\
& +\mathcal{L} \int_{T_{0}}^{t}\left(\mathcal{M}^{\int_{\sigma(\tau)}^{T_{2 i}}|\gamma(s)| d s}\right) \prod_{s \in[\sigma(\tau), t) \cap \mathbb{T}^{s}} \mathcal{M}\|\varepsilon(\tau)\| \Delta \tau .
\end{aligned}
$$

Multiplying both sides of this inequality by

$$
\left(\mathcal{M}^{-\sum_{j=1}^{i} \int_{T_{2 j-1}}^{T_{2 j}}|\gamma(s)| d s}\right) \prod_{s \in\left(T_{0}, t\right) \cap \mathbb{T}^{s}} \mathcal{M}^{-1},
$$

we obtain

$$
\begin{aligned}
& \|\varepsilon(t)\|\left(\mathcal{M}^{-\sum_{j=1}^{i} \int_{T_{2 j-1}}^{T_{2 j}}|\gamma(s)| d s}\right) \prod_{s \in\left(T_{0}, t\right) \cap \mathbb{T}^{s}} \mathcal{M}^{-1} \\
& \quad \leq\left\|\varepsilon_{T_{0}}\right\| \mathcal{M}+\int_{T_{0}}^{t} \mathcal{L}\|\varepsilon(\tau)\|\left(\mathcal{M}^{-\left(\sum_{j=1}^{i} \int_{T_{2 j-1}}^{T_{2 j}}|\gamma(s)| d s+\int_{T_{2 i}}^{\sigma(\tau)}|\gamma(s)| d s\right)}\right) \prod_{s \in\left(T_{0}, \tau\right] \cap \mathbb{T}^{s}} \mathcal{M}^{-1} \Delta \tau .
\end{aligned}
$$

Since $\mathcal{M}^{-\int_{T_{0}}^{T_{0}}|\gamma(s)| d s}=1$,

$$
\begin{aligned}
\|\varepsilon(t)\|\left(\mathcal{M}^{-\sum_{j=1}^{i} \int_{T_{2 j-1}}^{T_{2 j}}|\gamma(s)| d s}\right) \prod_{s \in\left(T_{0}, t\right) \cap \mathbb{T}^{s}} \mathcal{M}^{-1} \\
\leq\left\|\varepsilon_{T_{0}}\right\| \mathcal{M}^{-\int_{T_{0}}^{T_{0}}|\gamma(s)| d s} \cdot \mathcal{M} \\
\quad+\int_{T_{0}}^{t} \mathcal{L}\|\varepsilon(\tau)\|\left(\mathcal{M}^{-\left(\sum_{j=1}^{i} \int_{T_{2 j-1}}^{T_{2 j}}|\gamma(s)| d s+\int_{T_{2 i}}^{\sigma(\tau)}|\gamma(s)| d s\right)}\right) \prod_{s \in\left(T_{0}, \tau\right] \cap \mathbb{T}^{s}} \mathcal{M}^{-1} \Delta \tau .
\end{aligned}
$$


Using $\sigma(\tau)=\tau$, we get

$$
\begin{aligned}
& \|\varepsilon(t)\|\left(\mathcal{M}^{-\sum_{j=1}^{i} \int_{T_{2 j-1}}^{T_{2 j}}|\gamma(s)| d s}\right) \prod_{s \in\left(T_{0}, t\right) \cap \mathbb{T}^{s}} \mathcal{M}^{-1} \\
& \leq\left\|\varepsilon_{T_{0}}\right\| \mathcal{M}^{-\int_{T_{0}}^{T_{0}}|\gamma(s)| d s} \cdot \mathcal{M} \\
& \quad+\int_{T_{0}}^{t} \mathcal{L}\|\varepsilon(\tau)\|\left(\mathcal{M}^{-\left(\sum_{j=1}^{i} \int_{T_{2 j-1}}^{T_{2 j}}|\gamma(s)| d s+\int_{T_{2 i}}^{\tau}|\gamma(s)| d s\right)}\right) \prod_{s \in\left(T_{0}, \tau\right] \cap \mathbb{T}^{s}} \mathcal{M}^{-1} \Delta \tau .
\end{aligned}
$$

By Lemma 1 this leads to the inequality

$$
\|\varepsilon(t)\|\left(\mathcal{M}^{-\sum_{j=1}^{i} \int_{T_{2 j-1}}^{T_{2 j}}|\gamma(s)| d s}\right) \prod_{s \in\left(T_{0}, t\right) \cap \mathbb{T}^{s}} \mathcal{M}^{-1} \leq\left\|\varepsilon_{T_{0}}\right\| \mathcal{M} e_{\mathcal{L} \mathcal{M}^{-1}}\left(t, T_{0}\right) .
$$

Using Lemma 4, we have

$$
\begin{aligned}
& \|\varepsilon(t)\|\left(\mathcal{M}^{-\sum_{j=1}^{i} \int_{T_{2 j-1}}^{T_{2 j}}|\gamma(s)| d s}\right) \prod_{s \in\left(T_{0}, t\right) \cap \mathbb{T}^{s}} \mathcal{M}^{-1} \\
& \quad \leq\left\|\varepsilon_{T_{0}}\right\| \mathcal{M}\left(e^{\mathcal{L} \mathcal{M}^{-1} \sum_{j=1}^{i}\left(T_{2 j}-T_{2 j-1}\right)}\right) \prod_{s \in\left[T_{0}, t\right) \cap \mathbb{T}^{s}}\left(1+\mu(s) \mathcal{L} \mathcal{M}^{-1}\right) .
\end{aligned}
$$

Hence

$$
\begin{aligned}
\|\varepsilon(t)\| \leq & \left\|\varepsilon_{T_{0}}\right\|\left(e^{\mathcal{L} \mathcal{M}^{-1} \sum_{j=1}^{i}\left(T_{2 j}-T_{2 j-1}\right)}\right) \\
& \cdot\left(\mathcal{M}^{\sum_{j=1}^{i} \int_{T_{2 j-1}}^{T_{2 j}}|\gamma(s)| d s}\right) \prod_{s \in\left[T_{0}, t\right) \cap \mathbb{T}^{s}}(\mathcal{M}+\mu(s) \mathcal{L}) .
\end{aligned}
$$

By (12) we have

$$
\begin{aligned}
\|\varepsilon(t)\| \leq & \left\|\varepsilon_{T_{0}}\right\|\left(e^{\mathcal{L} \mathcal{M}^{-1} \sum_{j=1}^{i}\left(T_{2 j}-T_{2 j-1}\right)}\right) \\
& \cdot\left(\mathcal{M}^{\sum_{j=1}^{i} \int_{T_{2 j-1}}^{T_{2 j}}|\gamma(s)| d s}\right) \prod_{s \in\left[T_{0}, t\right) \cap \mathbb{T}^{s}}\left(\mathcal{M}+\mu^{*} \mathcal{L}\right) .
\end{aligned}
$$

Analogously, for $t \in\left(T_{2 i+1}, T_{2 i+2}\right]$,

$$
\begin{aligned}
\|\varepsilon(t)\| \leq & \left\|\varepsilon_{T_{0}}\right\|\left(e^{\mathcal{L} \mathcal{M}^{-1} \sum_{j=1}^{i}\left(T_{2 j}-T_{2 j-1}\right)}\right) \\
& \cdot\left(\mathcal{M}^{\sum_{j=1}^{i} \int_{T_{2 j-1}}^{T_{2 j}}|\gamma(s)| d s+\int_{T_{2 i+1}}^{t}|\gamma(s)| d s}\right) \prod_{s \in\left[T_{0}, T_{2 i+1}\right) \cap \mathbb{T}^{s}}\left(\mathcal{M}+\mu^{*} \mathcal{L}\right) .
\end{aligned}
$$

Set

$$
\begin{aligned}
& \operatorname{sum}(i):=\sum_{j=1}^{i}\left(\mathcal{L} \mathcal{M}^{-1}\left(T_{2 j}-T_{2 j-1}\right)+\ln \mathcal{M} \int_{T_{2 j-1}}^{T_{2 j}}|\gamma(s)| d s\right), \\
& e_{d}^{*}\left(t, T_{0}\right):=e^{\operatorname{sum}(i)} \prod_{s \in\left[T_{0}, t\right) \cap \mathbb{T}^{s}}\left(\mathcal{M}+\mu^{*} \mathcal{L}\right) \quad \text { for } t \in\left(T_{2 i}, T_{2 i+1}\right]
\end{aligned}
$$




$$
e_{d}^{* *}\left(t, T_{0}\right):=e^{\operatorname{sum}(i)+\int_{T_{2 i+1}}^{t}|\gamma(s)| d s} \prod_{s \in\left[T_{0}, T_{2 i+1}\right) \cap \mathbb{T}^{s}}\left(\mathcal{M}+\mu^{*} \mathcal{L}\right) \quad \text { for } t \in\left(T_{2 i+1}, T_{2 i+2}\right]
$$

for $i \in \mathbb{N}_{0}$, and

$$
e_{d}\left(t, T_{0}\right):= \begin{cases}e_{d}^{*}\left(t, T_{0}\right) & \text { for } t \in\left(T_{2 i}, T_{2 i+1}\right] \\ e_{d}^{* *}\left(t, T_{0}\right) & \text { for } t \in\left(T_{2 i+1}, T_{2 i+2}\right]\end{cases}
$$

By (13) and (14) inequalities (15) and (16) imply the thesis.

Corollary 1 Let conditions (7)-(9) and (12) be satisfied. Moreover, suppose that

for any $t \in \mathbb{T}^{s}$, there exists $\tilde{t} \in \mathbb{T}^{d}$ such that $\tilde{t}>t$ and

for any $t \in \mathbb{T}^{d}$, there exists $\tilde{t} \in \mathbb{T}^{s}$ such that $\tilde{t}>t$,

$$
\mathcal{M}+\mu^{*} \mathcal{L}<1,
$$

and

$$
\lim _{i \rightarrow \infty} e^{\mathrm{sum}(i)}<\infty
$$

Then equation (5) is exponentially stable.

Proof By (17) we get that $t \rightarrow \infty$ iff $i \rightarrow \infty$. Since $0<\mathcal{M}+\mu^{*} \mathcal{L}<1$ and $\mathcal{M} \in(0,1)$, by properties of the functions $\mathcal{M}^{t}$ and $e^{t}$ condition (19) implies conditions (13) and (14). Hence assumptions of Theorem 1 are satisfied. So, the statement holds.

Example 4 Let

$$
\mathbb{T}=\bigcup_{i=3}^{\infty}\left[\frac{i}{2}, \frac{i}{2}+\frac{1}{i^{3}}\right]
$$

Here $\mathbb{T}^{d}=\bigcup_{i=3}^{\infty}\left[\frac{i}{2}, \frac{i}{2}+\frac{1}{i^{3}}\right)$ and $\mathbb{T}^{s}=\left\{\frac{i}{2}+\frac{1}{i^{3}}: i \in \mathbb{N}, i \geq 3\right\}$,

$$
T_{0}=T_{1}=1.500, \quad T_{2} \approx 1.537, \quad T_{3}=2.000, \quad \ldots, \quad \mu^{*}=0.500 .
$$

Moreover, let

$$
\begin{array}{ll}
f(t, x)=0.100 \frac{x}{t^{2}}, & \gamma(t) \equiv 0.500(t-1), \\
A=\left[\begin{array}{llll}
0 & 0 & 1 & 1 \\
0 & 0 & 0 & 0 \\
1 & 0 & 0 & 1 \\
1 & 0 & 1 & 0
\end{array}\right], \quad D=\left[\begin{array}{llll}
0 & 0 & 0 & 0 \\
0 & 3 & 0 & 0 \\
0 & 0 & 1 & 0 \\
0 & 0 & 0 & 1
\end{array}\right]
\end{array}
$$

in equation (5) (see Fig. 1). 
Figure 1 The topology of the leader-following multi-agent system under the undirected graph

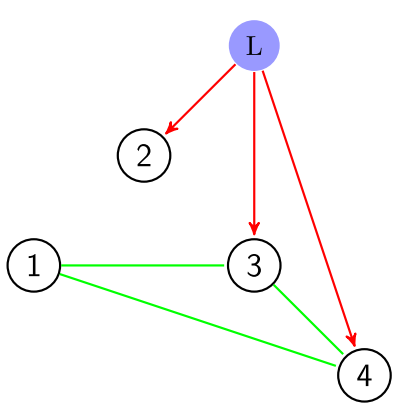

Hence

$$
L=\left[\begin{array}{cccc}
2 & 0 & -1 & -1 \\
0 & 0 & 0 & 0 \\
-1 & 0 & 2 & -1 \\
-1 & 0 & -1 & 2
\end{array}\right] \text { and } B=\left[\begin{array}{cccc}
2 & 0 & -1 & -1 \\
0 & 3 & 0 & 0 \\
-1 & 0 & 3 & -1 \\
-1 & 0 & -1 & 3
\end{array}\right]
$$

We have $\mathcal{L}=0.100, \lambda_{1}=2-\sqrt{2}, \lambda_{2}=2, \lambda_{3}=3$, and $\lambda_{4}=2+\sqrt{2}$. From this it follows that $\lambda_{\text {min }}=\min \left\{\lambda_{1}, \lambda_{2}, \lambda_{3}, \lambda_{4}\right\} \approx 0.585$ and

$$
\max \left\{1-0.462 \cdot 0.250 \cdot 0.585, e^{-0.585}\right\} \leq \max \{0.933,0.557\}=0.933=: \mathcal{M}
$$

From the above we get

$$
\prod_{s \in \mathbb{T}^{s}}\left(\mathcal{M}+\mu^{*} \mathcal{L}\right) \approx \prod_{s \in \mathbb{T}^{s}} 0.983=0
$$

and

$$
\lim _{i \rightarrow \infty} e^{\operatorname{sum}(i)} \approx \lim _{i \rightarrow \infty} e^{\sum_{j=3}^{i}\left(0.108 j^{-3}-0.070 \cdot 0.250\left(i^{-2}-2 i^{-3}+i^{-6}\right)\right)}<\infty .
$$

All assumptions of Corollary 1 are satisfied, and thus equation (5) is exponentially stable. System (2)-(3) achieves consensus exponentially.

In Example 4 we have

$$
\lim _{i \rightarrow \infty} e^{\mathcal{L} \mathcal{M}^{-1} \sum_{j=1}^{i}\left(T_{2 j}-T_{2 j-1}\right)} \approx \lim _{i \rightarrow \infty} e^{0.108 \sum_{j=3}^{i} j^{-3}}<\infty
$$

but this condition is not required for the exponential stability of (5) (see Example 5).

Remark 2 If conditions (7)-(9), (12), and (18) are satisfied and if

$$
\gamma(t) \equiv \gamma \in \mathbb{R}
$$

and

$$
\mathcal{L} \mathcal{M}^{-1}+\gamma \ln \mathcal{M}<0
$$

then equation (5) is exponentially stable. 
Proof If condition (21) holds, then

$$
\begin{aligned}
\operatorname{sum}(i) & =\sum_{j=1}^{i}\left(\mathcal{L} \mathcal{M}^{-1}\left(T_{2 j}-T_{2 j-1}\right)+\gamma\left(T_{2 j}-T_{2 j-1}\right) \ln \mathcal{M}\right) \\
& =\left(\mathcal{L} \mathcal{M}^{-1}+\gamma \ln \mathcal{M}\right) \sum_{j=1}^{i}\left(T_{2 j}-T_{2 j-1}\right) .
\end{aligned}
$$

By (22) we see that $\operatorname{sum}(i)<0$ for any $i \in \mathbb{N}$ and $e^{\text {sum(i) }}$ is a positive decreasing function of the variable $i \in \mathbb{N}$. Here $\prod_{s \in \mathbb{T}^{s}}\left(\mathcal{M}+\mu^{*} \mathcal{L}\right)$ and $e^{\text {sum(i) }}$ for any $i \in \mathbb{N}$ are bounded. If the cardinality of set $\mathbb{T}^{s}$ is infinity, then $\lim _{s \rightarrow \infty} \prod_{s \in \mathbb{T}^{s}}\left(\mathcal{M}+\mu^{*} \mathcal{L}\right)=0$. If the cardinality of set $\mathbb{T}^{d}$ is infinity, then $\lim _{i \rightarrow \infty} e^{\operatorname{sum}(i)}=0$. Thus, by Theorem 1 , we obtain the statement.

Example 5 Let

$$
\mathbb{T}=\bigcup_{i=3}^{\infty}\left[\frac{i}{2}, \frac{i}{2}+\frac{1}{i}\right]
$$

Here $\mathbb{T}^{d}=\bigcup_{i=3}^{\infty}\left[\frac{i}{2}, \frac{i}{2}+\frac{1}{i}\right)$ and $\mathbb{T}^{s}=\left\{\frac{i}{2}+\frac{1}{i}: i \in \mathbb{N}, i \geq 3\right\}$,

$$
\begin{aligned}
& T_{0}=T_{1}=1.500, \quad T_{2} \approx 1.833, \quad T_{3}=2.000 \\
& \mu(t)=\frac{1}{2}-\frac{t}{2}+\frac{1}{2} \sqrt{t^{2}-2} \quad \text { for } t \in \mathbb{T}^{s}, \quad \mu^{*}=0.500
\end{aligned}
$$

Moreover, let

$$
f(t, x)=0.250 \frac{\sin x}{t^{2}}, \quad \gamma(t) \equiv 2.000
$$

and let the matrix $B$ be given by (20) in equation (5). Then $\mathcal{L}=0.250, \lambda_{\min } \approx 0.585$, and

$$
\max \left\{1-0.333 \cdot 2.000 \cdot 0.585, e^{-0.585}\right\} \leq \max \{0.390,0.557\}=0.557=: \mathcal{M}
$$

Finally,

$$
\mathcal{L} \mathcal{M}^{-1}+\gamma \ln \mathcal{M} \approx 0.449-1.170=-0.721<0 .
$$

All assumptions of Remark 2 hold, and thus equation (5) is exponentially stable.

In Example 5 we have

$$
\lim _{i \rightarrow \infty} e^{\mathcal{L} \mathcal{M}^{-1} \sum_{j=1}^{i}\left(T_{2 j}-T_{2 j-1}\right)} \approx \lim _{i \rightarrow \infty} e^{0.449 \sum_{j=1}^{i} \frac{1}{i}}=\infty
$$

although system (2)-(3) achieves consensus exponentially. 
Corollary 2 If conditions (7)-(9) and (18) are satisfied and if

$$
\sum_{i=0}^{\infty}\left(T_{2 i+2}-T_{2 i+1}\right)<\infty
$$

then equation (5) is exponentially stable.

Proof Since (23) holds,

$$
e^{\mathrm{sum}(i)}=\text { constant. }
$$

Hence, recalling that the cardinality of the set $\mathbb{T}^{s}$ is infinity, by (18) we obtain

$$
\begin{aligned}
& \lim _{i \rightarrow \infty} e^{\mathrm{sum}(i)} \prod_{s \in\left[T_{0}, T_{2 i}\right) \cap \mathbb{T}^{s}}\left(\mathcal{M}+\mu^{*} \mathcal{L}\right) \\
& =\lim _{i \rightarrow \infty} c^{*}\left(\prod_{s \in\left[T_{0}, T_{2 i}\right) \cap \mathbb{T}^{s}}\left(\mathcal{M}+\mu^{*} \mathcal{L}\right)\right) \\
& =c^{*} \prod_{s \in \mathbb{T}^{s}}\left(\mathcal{M}+\mu^{*} \mathcal{L}\right)=0
\end{aligned}
$$

where $c^{*}=e^{\mathrm{sum}(i)}$.

For two possible cases of carrying out of assumption (23), see Examples 4 and 7.

Theorem 1 generalizes Theorem 2 [14]. In the following example we present an equation on time scale for which Theorem 2 [14] cannot be applied, but our Corollary 2 of Theorem 1 can be.

Example 6 Let

$$
\mathbb{T}=\{i: i \in \mathbb{N}\} \cup\left\{i+\frac{1}{j+1}: i, j \in \mathbb{N}, j \geq 2\right\}
$$

Here $\mathbb{T}^{d}=\{i: i \in \mathbb{N}\}$ and $\mathbb{T}^{s}=\left\{i+\frac{1}{j+1}: i, j \in \mathbb{N}, j \geq 2\right\}$

$$
T_{0}=1, \quad T_{1}=\infty, \quad \mu(t)=\frac{(t-i)^{2}}{1+t-i} \quad \text { for } t \in \mathbb{T}^{s}, \quad \mu^{*}=0.500
$$

Set $f(t, x)=0.250 x$,

$$
\gamma(t)= \begin{cases}\frac{1}{\mu(t)} & \text { for } t \in \mathbb{T}^{s} \\ 0 & \text { for } t \in \mathbb{T}^{d}\end{cases}
$$

and $B$ is given by (20) in equation (5). We have $\mathcal{L}=0.250, \lambda_{\min }=0.585$, and

$$
\max \left\{1-1 \cdot 0.585, e^{-0.585}\right\} \leq \max \{0.515,0.557\}=0.557=: \mathcal{M} .
$$


Hence

$$
\prod_{s \in \mathbb{T}^{s}}\left(\mathcal{M}+\mu^{*} \mathcal{L}\right) \approx \prod_{s \in \mathbb{T}^{s}} 0.682=0
$$

All assumptions of Corollary 2 are satisfied, and thus equation (5) is exponentially stable.

Since $\liminf _{t \rightarrow \infty} \mu(t)=0$, the results obtained in [14] cannot be applied.

The following examples show two different situations concerning time scale in which condition (23) is satisfied. In the first example, $\mathbb{T}^{d}$ is a bounded set. In the second one, $\mathbb{T}^{d}$ is unbounded.

\section{Example 7 Let}

$$
\mathbb{T}=[1,2] \cup[3,7] \cup\{n: n \in \mathbb{N}, n \geq 8\}
$$

Here $\mathbb{T}^{d}=[1,2] \cup[3,7]$ is bounded, and $\mathbb{T}^{s}=\{n: n \in \mathbb{N}, n \geq 8\}$. We see that

$$
\begin{aligned}
& T_{0}=1, \quad T_{1}=T_{0}=1, \quad T_{2}=2, \quad T_{3}=3, \\
& T_{4}=7, \quad T_{5}=8, \quad T_{6}=\infty, \\
& \mu(t)=1 \quad \text { for } t \in \mathbb{T}^{s}, \quad \mu^{*}=1 .
\end{aligned}
$$

Let also

$$
f(t, x)=\frac{1}{4 \sqrt{t}} \sin x, \quad \gamma(t)=\cos t+2,
$$

and let the matrix $B$ be given by (20) in equation (5). Then $\mathcal{L}=0.250, \lambda_{\min }=0.585$ and

$$
\mathcal{M}:=\max \left\{1-1 \cdot 1 \cdot 0.585, e^{-0.585}\right\} \approx 0.557<1 .
$$

As a consequence,

$$
\prod_{s \in \mathbb{T}^{s}}\left(\mathcal{M}+\mu^{*} \mathcal{L}\right) \approx \prod_{s \in \mathbb{T}^{s}} 0.807=0
$$

All assumptions of Corollary 2 are satisfied, and thus equation (5) is exponentially stable. This means that the multi-agent system (2)-(3) achieves the leader-following consensus exponentially.

Example 8 Let

$$
\mathbb{T}=\bigcup_{i=3}^{\infty}\left[\frac{i}{2}+\frac{1}{i+1}, \frac{i}{2}+\frac{1}{i}\right]
$$

Here either $\mathbb{T}^{d}=\bigcup_{i=3}^{\infty}\left[\frac{i}{2}+\frac{1}{i+1}, \frac{i}{2}+\frac{1}{i}\right)$ or $\mathbb{T}^{s}=\left\{\frac{i}{2}+\frac{1}{i}: i \in \mathbb{N}, i \geq 3\right\}$ is an unbounded set. We see that

$$
T_{0}=T_{1}=1.750, \quad T_{2} \approx 1.833, \quad T_{3}=2.200, \quad T_{4}=2.250, \quad \ldots,
$$




$$
\mu(t)=\frac{1}{2}-\frac{2}{\left(t+\sqrt{t^{2}-2}\right)\left(2+t+\sqrt{t^{2}-2}\right)} \quad \text { for } t \in \mathbb{T}^{s}, \quad \mu^{*}=0.500
$$

Moreover,

$$
f(t, x)=\frac{x}{4 t}, \quad \gamma(t)=\frac{1}{4} t^{2},
$$

and the matrix $B$ is given by (20) in equation (5). Then $\mathcal{L}=0.250, \lambda_{\min }=0.585$, and

$$
\max \left\{1-0.366 \cdot 0.765 \cdot 0.585, e^{-0.585}\right\}<0.836=: \mathcal{M}
$$

Hence

$$
\prod_{s \in \mathbb{T}^{s}}\left(\mathcal{M}+\mu^{*} \mathcal{L}\right) \approx \prod_{s \in \mathbb{T}^{s}} 0.961=0
$$

All assumptions of Corollary 2 are satisfied, and thus equation (5) is exponentially stable. System (2)-(3) achieves consensus exponentially.

Notice that in Example 8 we have

$$
\lim _{i \rightarrow \infty} e^{\mathcal{L} \mathcal{M}^{-1} \sum_{j=1}^{i}\left(T_{2 j}-T_{2 j-1}\right)}=\lim _{i \rightarrow \infty} e^{0.299 \sum_{j=1}^{i} \frac{1}{i(i+1)}}=e^{0.299}<\infty .
$$

Remark 3 If conditions (7)-(9) are satisfied,

$$
\sum_{j=1}^{\infty} \int_{T_{2 j-1}}^{T_{2 j}}|\gamma(s)| d s<\infty,
$$

and

$$
\lim _{i \rightarrow \infty} e^{\mathcal{L} \mathcal{M}^{-1} \sum_{j=1}^{i}\left(T_{2 j}-T_{2 j-1}\right)} \cdot \prod_{s \in\left[T_{0}, T_{2 i}\right) \cap \mathbb{T}^{s}}\left(\mathcal{M}+\mu^{*} \mathcal{L}\right)=0
$$

then equation (5) is exponentially stable.

\section{(See Example 4.)}

Remark 4 Let conditions (7)-(9) be satisfied. If the cardinality of the set $\mathbb{T}^{s}$ is finite and $\operatorname{sum}(i)<0$ for any $i \in \mathbb{N}$, then equation (5) is exponentially stable.

Example 9 Let

$$
\mathbb{T}=\{1\} \cup\{11\} \cup[12, \infty)
$$

Here $\mathbb{T}^{d}=[12, \infty)$ is an unbounded set, whereas $\mathbb{T}^{s}=\{1\} \cup\{11\}$ is bounded, and

$$
T_{0}=1, \quad T_{1}=12, \quad T_{2}=\infty, \quad \mu(1)=10, \quad \mu(11)=1, \quad \mu^{*}=10 .
$$


Let

$$
f(t, x)=0.1 x, \quad \gamma(t)=1
$$

and let the matrix $B$ be given by (20) in equation (5). Then $\mathcal{L}=0.100, \lambda_{\min } \approx 0.585$, and

$$
\max \left\{1-0.585, e^{-0.585}\right\}<0.557=: \mathcal{M}
$$

Hence

$$
\operatorname{sum}(i) \approx 0.180\left(T_{2}-T_{1}\right)-0.585 \int_{T_{1}}^{T_{2}} d s=-0.405\left(T_{2}-T_{1}\right)=-\infty<0 .
$$

All assumptions of Remark 4 are satisfied, and thus equation (5) is exponentially stable.

Notice that in Example 9 condition (18) does not hold.

\section{Acknowledgements}

Not applicable.

\section{Funding}

Ewa Schmeidel was supported by the Polish National Science Center grant on the basis of decision

DEC-2014/15/B/ST7/05270. Urszula Ostaszewska and Małgorzata Zdanowicz have been supported by the Polish Ministry of Science and Higher Education under a subsidy for maintaining the research potential of the Faculty of Mathematics and Informatics, University of Bialystok.

\section{Availability of data and materials}

Not applicable.

\section{Competing interests}

The authors declare that they have no competing interests.

\section{Authors' contributions}

The main idea of this paper comes from Ewa Schmeidel. Proofs of theorems and examples are the joint work of all coauthors. All authors read and approved the final manuscript.

\section{Publisher's Note}

Springer Nature remains neutral with regard to jurisdictional claims in published maps and institutional affiliations.

Received: 20 April 2019 Accepted: 28 May 2019 Published online: 13 June 2019

\section{References}

1. DeGroot, M.H.: Reaching a consensus. J. Am. Stat. Assoc. 69, 118-121 (1974). https://doi.org/10.1080/01621459

2. Krause, U.: A discrete nonlinear and non-autonomous model of consensus formation. In: Communications in Difference Equations. ICDEA, vol. 1998. Gordon \& Breach, Poznań (2000). https://doi.org/10.1109//YCE.2015.7180798

3. Hegselmann, R., Krause, U.: Opinion dynamics and bounded confidence: models, analysis, and simulation. J. Artif. Soc. Soc. Simul. 5, 1-33 (2002)

4. Jadbabaie, A., Lin, J., Morse, A.S.: Coordination of groups of mobile autonomous agents using nearest neighbor rules. IEEE Trans. Autom. Control 48(6), 988-1001 (2003). https://doi.org/10.1109/TAC.2003.812781

5. Blondel, V.D., HendrickX, J.M., Tsitsikli, J.N.: On Krause's multi-agent consensus model with state-dependent connectivity. IEEE Trans. Autom. Control 54(11), 2586-2597 (2009). https://doi.org/10.1109/TAC.2009.2031211

6. Blondel, V.D., Hendrickx, J.M., Tsitsikli, J.N.: Continuous-time average-preserving opinion dynamics with opinion-dependent communications. SIAM J. Control Optim. 18(8), 5214-5240 (2010). https://doi.org/10.1137/090766188

7. Girejko, E., Machado, L., Malinowska, A.B., Martins, N.: Krause's model of opinion dynamics on isolated time scales. Math. Methods Appl. Sci. 39(18), 5302-5314 (2016). https://doi.org/10.1002/mma.3916

8. Girejko, E., Malinowska, A.B., Schmeidel, E., Zdanowicz, M.: The emergence on isolated time scales. In: 21st International Conference on Methods and Models in Automation and Robotics (MMAR), IEEExplore (2016). https://doi.org/10.1109/MMAR.2016.7575317

9. Cucker, F., Smale, S.: On the mathematics of emergence. Jpn. J. Math. 2(1), 197-227 (2007) https://doi.org/10.1007/s11537-007-0647-x 
10. Cucker, F., Smale, S.: Emergent behavior in flocks. IEEE Trans. Autom. Control 52(7), 852-862 (2007). https://doi.org/10.1109/TAC.2007.895842

11. Girejko, E., Machado, L., Malinowska, A.B., Martins, N.: On consensus in the Cucker-Smale type model on isolated time scale. Discrete Contin. Dyn. Syst., Ser. B 11(1), 77-89 (2018). https://doi.org/10.3934/dcdss.2018005

12. Wang, Y., Cheng, L., Wang, H., Hou, Z.G., Tan, M., Yu, H.: Leader-following consensus of discrete-time linear multi-agent systems with communication noises. In: 34th Control Conference (CCC), Hangzhou, China. Lecture Notes in Electrical Engineering, vol. 407. IEEE, New York (2015). https://doi.org/10.1109/ChiCC.2015.7260748

13. Ostaszewska, U., Schmeidel, E., Zdanowicz, M.: Leader-following consensus on discrete time scales. In: ICNAAM 2017, Thessaloniki, Greece. AlP Conference Proceedings. American Institute of Physics, New York (2018). https://doi.org/10.1063/1.5044162

14. Ostaszewska, U., Schmeidel, E., Zdanowicz, M.: Emergence of consensus of multi-agents systems on time scales. Miskolc Math. Notes (to appear)

15. Aulbach, B., Hilger, S.: A unified approach to continuous and discrete dynamics. In: Qualitative Theory of Differential Equations (Szeged, 1988). Colloq. Math. Soc. Jámos Bolyai, vol. 53. North-Holland, Amsterdam (1990)

16. Bohner, M., Peterson, A.: Dynamic Equations on Time Scales. Birkhäuser, Boston (2001). https://doi.org/10.1109/IYCE.2015.7180798

17. Bohner, M., Peterson, A.: Advances in Dynamic Equations on Time Scales. Birkhäuser, Boston (2003)

18. Yu, Z., Jiang, H., Hu, C.: Leader-following consensus of fractional-order multi-agent systems under fixed topology. Neurocomputing 149, 613-620 (2015). https://doi.org/10.1016/j.neucom.2014.08.013

19. Schmeidel, E.: The existence of consensus of a leader-following problem with Caputo fractional derivative. Opusc Math. 39(1), 77-89 (2019). https://doi.org/10.7494/OpMath.2019.39.1.77

20. Berezansky, L., Migda, M., Schmeidel, E.: Some stability conditions for scalar Volterra difference equations. Opusc. Math. 36(4), 459-470 (2016). https://doi.org/10.7494/OpMath.2016.36.4.459

21. Elaydi, S.N.: An Introduction to Difference Equations. Springer, New York (2005)

22. Peterson, A., Raffoul, Y.N.: Exponential stability of dynamic equations on time scales. Adv. Differ. Equ. 2005, 858671 (2005). https://doi.org/10.1155/ADE.2005.133

23. Bodine, S., Lutz, D.A.: Exponential functions on time scales: their asymptotic behavior and calculation. Dyn. Syst. Appl. $12(1-2), 23-43(2003)$

\section{Submit your manuscript to a SpringerOpen ${ }^{\circ}$ journal and benefit from:}

- Convenient online submission

- Rigorous peer review

- Open access: articles freely available online

- High visibility within the field

- Retaining the copyright to your article

Submit your next manuscript at $\gg$ springeropen.com 